\title{
A numerical analysis of the influence of the liquid depth on two-dimensional Faraday waves
}

\author{
S. Ubal, M. D. Giavedoni, and F. A. Saita ${ }^{\text {a) }}$ \\ Instituto de Desarrollo Tecnológico para la Industria Química, \\ CONICET-Universidad Nacional del Litoral, Güemes 3450-3000 Santa Fe, Argentina
}

(Received 24 September 2002; accepted 13 June 2003; published 5 September 2003)

\begin{abstract}
In this work a numerical analysis of two-dimensional Faraday waves is presented. This study is based on direct numerical simulation of Navier-Stokes and continuity equations with appropriate boundary conditions. Stability maps on the $(F-\alpha)$ plane for viscous liquid layers with equilibrium depths between $5 \times 10^{-5} \mathrm{~m}$ and $10^{-5} \mathrm{~m}$ are presented; comparisons are made with the linear stability predictions obtained with Benjamin and Ursell's model for an inviscid fluid and with Kumar and Tuckerman's model for a viscous fluid. Regions in which time-periodic solutions are no longer obtained and nonlinear effects are relevant, and are also delimited and analyzed: in these zones the disintegration of the free surface into drops may take place. (c) 2003 American Institute of Physics. [DOI: 10.1063/1.1601220]
\end{abstract}

\section{INTRODUCTION}

When a container filled with a liquid is subject to a vertical oscillation, waves may be formed at the gas-liquid interface. For given physicochemical properties of the liquid, the required conditions under which a wavy interface is developed depend on the frequency and the amplitude of the imposed vibration, the depth of the liquid layer and the geometry of the container.

The analysis of this system is a phenomenon closely related to the storage and transportation of liquids when the frequency of the external vibration is low, and to the atomization of liquids, at large frequencies of the imposed acceleration. The atomization of liquids is important in mass transfer operations in which the mean size of the drops formed should be very small. Because the mean diameter of the drops ejected from the free surface is proportional to the inverse of the excitation frequency, it is possible to produce very small drops if the period of the imposed oscillation is small enough.

This problem was first investigated by Faraday ${ }^{1}$ who also reported that the frequency of the surface waves, today known as Faraday waves, is equal to one half the frequency of the external forcing. Many years later, Benjamin and Ursell $^{2}$ explained the phenomena reported by Faraday analyzing the linearized hydrodynamic inviscid problem. They derived a set of Mathieu's equations and showed that resonance is responsible for the wavy motion. They also concluded that the free surface is always unstable whenever the ratio between any of the natural frequencies of the system and the external one is equal to $n / 2(n=1,2,3, \ldots)$, even for amplitudes of the imposed acceleration infinitesimally small. This unrealistic result is a consequence of the ideal behavior assumed.

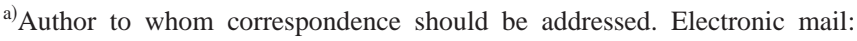
fasaita@ceride.gov.ar
}

Ockendon and Ockendon ${ }^{3}$ extended the analysis presented by Benjamin and Ursell to small but finite perturbations and included nonlinear terms. Miles ${ }^{4}$ proposed an averaged Lagrangian approach from which a weakly nonlinear model can be derived. He introduced additional linear terms into the evolution equations to approximate the damping effects produced by viscous dissipation at the solid boundaries and at the free surface when it is covered by a viscoelastic surface film, and by capillary hysteresis associated with the presence of contact lines. ${ }^{5}$

These works were followed by others in which weakly nonlinear models were discussed. A review of these analyses was presented by Miles and Henderson ${ }^{6}$ in 1990 and by Miles $^{7}$ in 1993.

Kumar and Tuckerman ${ }^{8}$ were the first to derive and to solve the linear stability problem for the interface of two viscous fluids subject to a vertical oscillation, based on the complete hydrodynamic problem; that is, Navier-Stokes and continuity equations. They show that under the effect of viscosity the hydrodynamic system cannot be reduced to a set of Mathieu equations with a linear damping term that is the result of the phenomenological approach usually adopted in the literature. A few years later, Cerda and Tirapegui ${ }^{9}$ restated the problem analyzed by Kumar and Tuckerman; the expression obtained for the amplitude of the free surface (an integro-differential equation) takes into account viscous effects in the bulk, near the bottom wall of the container and at the free surface. In their analysis, these authors show that their equation can be reduced to a Mathieu equation for a highly viscous fluid; however, it differs from that derived by Benjamin and Ursell ${ }^{2}$ for the inviscid case.

Although the literature related to Faraday waves is considerable, there are very few studies in which the numerical solution of the complete hydrodynamic problem is considered. In fact, numerical works on two-dimensional Faraday waves have been presented very recently by Wright et al. ${ }^{10}$ and Murakami and Chikano. ${ }^{11}$ 
Wright et al. ${ }^{10}$ analyzed the standing periodic waves formed at the interface of two ideal fluids. They employed two numerical methods, the boundary integral method when the density of one of the fluids is negligible and a vortex sheet method, otherwise. In their analysis, both fluids were incompressible and they extended to infinity; the effect of viscosity was considered including an extra term in the Euler equation.

Murakami and Chikano ${ }^{11}$ used the SMAC method to solve the governing equations (Navier-Stokes and continuity equations) with appropriate boundary conditions, once they were transformed into a boundary-time-dependent coordinate system. The values of the parameters of the system analyzed in that work were the same as those of the experiments reported by Lioubashevski et al. ${ }^{12}$ corresponding to a highly viscous fluid. Murakami and Chikano discussed the velocity fields of the two-dimensional standing waves developed near the onset of the instability; they also investigated numerically their stability to two-dimensional disturbances in order to reproduce the localized state reported by Lioubashevski et al. However, the uniform standing-waves proved to be stable, suggesting that the localized states are a threedimensional phenomenon.

In this work we study the evolution of a thin layer of a liquid subject to a vertical periodic acceleration of high frequency by numerically solving the full Navier-Stokes equations. Attention is restricted to the two-dimensional stability problem as in the abovementioned analysis.

The numerical technique employed is based on the Galerkin/finite element method combined with a suitable parametrization of the free surface (Kheshgi and Scriven ${ }^{13}$ ) that allows, at each time step, the simultaneous solution of the complete set of governing equations (Navier-Stokes and continuity) and boundary conditions.

The main goals of this work are: (i) to build the stability charts for various liquid depths in order to determine the influence of this parameter on the stability of the system; (ii) to validate our numerical predictions by comparing them with results obtained from linear stability analysis; e.g., those produced by Benjamin and Ursell for an ideal fluid and by Kumar and Tuckerman for a viscous one; (iii) to delimit the regions in which our numerical predictions are unstable and the motion of the free surface is so intense that standing waves are no longer present; (iv) to analyze the velocity fields in order to relate the viscous effects associated to wall friction in thin films to the minimum force required to destabilize the system. Point (iii) is important because it locates regions of the stability maps where drops may be produced and ejected from the free surface.

\section{MATHEMATICAL FORMULATION}

A Newtonian and incompressible liquid of viscosity $\mu$ and density $\rho$, is lying on a horizontal solid plate. The air above the liquid is regarded as inviscid, it exerts only normal stresses along the gas-liquid interface and its pressure is uniform everywhere. The system is isothermic and there are not surface active agents present; therefore all the physicochemical properties including the surface tension $(\sigma)$ are uniform

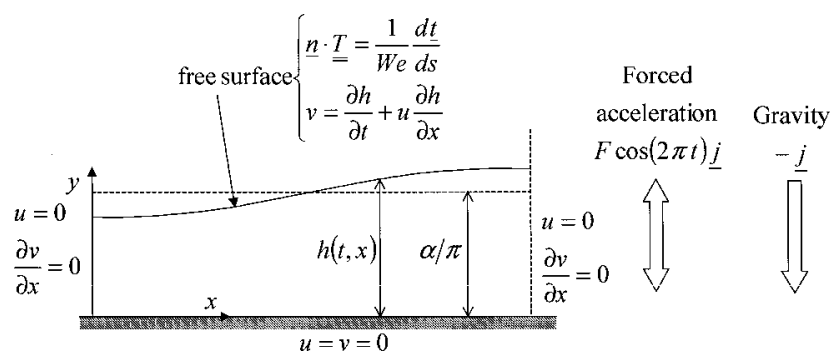

FIG. 1. Schematic representation of the flow domain, boundary conditions, and coordinate system adopted.

and constant. The liquid layer is extended on the horizontal plane $(x, z)$ and its equilibrium height measured along the $y$-coordinate is $H_{0}$. A vertical harmonic oscillation of amplitude $a_{0}$ and frequency $\omega$ is imposed to the system in order to destabilize the free surface; since the reference frame adopted (see Fig. 1) is attached to the solid wall the external acceleration is added to gravity acceleration.

In this work we consider only two-dimensional (2-D) motions of the liquid, then its height can be expressed in dimensionless form as $h(t, x)$.

Initially, a 2-D sinusoidal perturbation of amplitude $\varepsilon H_{0}$ and wave number $k$ is imposed, and the temporal evolution of this disturbance is followed. The extension of the domain in the $x$-direction is equal to one half the wavelength of the initial perturbation; therefore, the lateral boundaries are symmetry planes and the wavy motion developed is repeated at both sides of this domain.

Fluid motion is governed by Navier-Stokes and continuity equations. These expressions are made dimensionless by means of the following scales: $\pi / k=\pi H_{0} / \alpha$ for lengths, $2 \pi / \omega$ for time, $\omega H_{0} / 2 \alpha$ for the velocities and $\rho\left(\omega H_{0} / 2 \alpha\right)^{2}$ for pressure and stresses. Thus, the equations expressing conservation of momentum and mass are

$$
\begin{aligned}
\frac{\partial \mathbf{v}}{\partial t}+\mathbf{v} \cdot \nabla \mathbf{v}= & -\nabla p+\frac{1}{\operatorname{Re}} \nabla \cdot\left[\nabla \mathbf{v}+(\nabla \mathbf{v})^{T}\right] \\
& +\frac{1}{\mathrm{Fr}}[F \cos (2 \pi t)-1] \mathbf{j},
\end{aligned}
$$

$\nabla \cdot \mathbf{v}=0$,

where $\operatorname{Re}=\rho \omega \pi H_{0}^{2} / 2 \mu \alpha^{2}$ is the Reynolds number, Fr $=\omega^{2} H_{0} / 4 \pi g \alpha$ is the Froude number and $F=a_{0} \omega^{2} / g$ gives the ratio between the external imposed force and the gravitational force. The initial perturbation in dimensionless form is

$$
h(0, x)=\alpha / \pi[1+\varepsilon \sin (\pi x-\pi / 2)], \quad 0 \leqslant x \leqslant 1 .
$$

The boundary conditions required by Navier-Stokes equations are summarized in Fig. 1 where $u$ and $v$ are the $x$-and $y$-components of the velocity vector, respectively. At the bottom wall the nonslip condition is imposed while at the lateral planes, symmetry is required.

At the free surface, the adjacent gas phase exerts only normal stresses through its pressure which is chosen as the datum pressure and is arbitrarily set equal to zero; therefore, continuity of stresses at the interface is expressed as 


$$
\mathbf{n} \cdot \mathbf{T}=\frac{1}{\mathrm{We}} \frac{d \mathbf{t}}{d s}, \quad y=h(t, x),
$$

where $\mathbf{T}$ is the stress tensor on the liquid side of the interface, $\mathbf{n}$ is the outward pointing unit normal to the free surface and $\mathbf{t}$ is the unit tangent to the interface. We $=\pi \rho \omega^{2} H_{0}^{3} / 4 \sigma \alpha^{3}$ is the Weber number. Due to the hypotheses stated at the beginning of this section, surface tension is uniform and constant; therefore, the tangential component of the stresses at the free surface vanishes.

The free surface is a material surface because mass is not transferred through it. The expression of mass conservation is the kinematic condition that for a two-dimensional flow in the $(x, y)$ plane is

$$
v=\frac{\partial h}{\partial t}+u \frac{\partial h}{\partial x}, \quad y=h(t, x)
$$

The free surface requires boundary conditions; we impose symmetry conditions at $x=0$ and $x=1$.

\section{NUMERICAL TECHNIQUE}

The Galerkin/finite element method is used to obtain the spatial discrete form of the governing equations while the free surface location is traced with the aid of a suitable parametrization (Kistler and Scriven ${ }^{14}$ ).

The flow domain is tessellated into quadrilateral elements, the vertical sides of any element are spines with base points located at fixed values of the $x$-coordinate, while the shape and location of the horizontal sides depend on those of the free surface. Each quadrilateral element is mapped isoparametrically onto a unit square with coordinates $(\xi, \eta)$, $0 \leqslant \xi, \eta \leqslant 1$ by means of nine node biquadratic basis functions $\left(\Phi^{i}(\xi, \eta)\right)$,

$$
\begin{aligned}
& x(\xi, \eta)=\sum_{i=1}^{9} x^{i} \Phi^{i}(\xi, \eta), \\
& y(t, \xi, \eta)=\sum_{i=1}^{9} y^{i}(t) \Phi^{i}(\xi, \eta),
\end{aligned}
$$

where $\left(x^{i}, y^{i}(t)\right)$ are the nodal coordinates of the element. The above transformation applied to all the elements of the flow domain defines the computational domain in which the free surface is a coordinate line and it is approximated by the one-dimensional specialization of the biquadratic basis functions,

$$
y_{F S}=\sum_{i=1}^{3} h^{i}(t) \Phi^{i}(\xi, \eta=1) .
$$

In Eq. (8), $h^{i}$ are the coefficients of the free surface parametrization and each one of them represents the distance along a given spine from the $x$-axis to the gas-liquid interface.

Mixed interpolation is used to approximate the velocity and pressure fields; consequently,

$$
\mathbf{v}(\mathbf{x}, t)=\sum_{i=1}^{9} \mathbf{v}^{i}(t) \Phi^{i}(\xi, \eta),
$$

$$
p(\mathbf{x}, t)=\sum_{k=1}^{4} p^{k}(t) \Psi^{k}(\xi, \eta),
$$

where $\mathbf{v}^{i}(t)$ and $p^{k}(t)$ are the nodal values and $\Psi^{k}(\xi, \eta)$ are the four bilinear basis functions defined in the unit square.

The numerical scheme employed is similar to that proposed by Gresho et al. ${ }^{15}$ to analyze transient flows with the modifications introduced by Kheshgi and Scriven ${ }^{13}$ to adapt this technique to the solution of transient free surface flow problems. Very briefly, the governing Eqs. (1) and (2) are weighted with the basis functions employed to interpolate the velocity and pressure fields, respectively, and they are integrated in the flow domain; the kinematic expression [Eq. (5)] is weighted with the one-dimensional specialization of the biquadratic basis functions and it is integrated along the free surface. A set of nonlinear ordinary differential equations is thus obtained. To evaluate the time derivatives appearing in the residuals ( $\partial \mathbf{v} / \partial t$ and $\partial h / \partial t)$, one must take into account that the points of the mesh are moving; since in the $(\xi, \eta)$ plane the nodal coordinates remain unchanged, these derivatives can be though of as time derivatives at a fixed point in the computational domain. Then,

$$
\frac{\partial \mathbf{v}}{\partial t}=\dot{\mathbf{v}}-\dot{\mathbf{x}} \cdot \nabla \mathbf{v}, \quad \dot{\mathbf{v}}=\sum_{i} \frac{d \mathbf{v}^{i}}{d t} \Phi^{i}(\xi, \eta),
$$

where $\dot{\mathbf{x}}$ is the velocity of a point with fixed isoparametric coordinates which are evaluated from Eqs. (6) and (7).

The set of nonlinear ordinary differential equations is reduced to a set of nonlinear algebraic equations using the following finite difference approximation:

A trapezoidal rule corrector is used to approximate the time derivatives $(\dot{\mathbf{v}}, \dot{\mathbf{x}})$ in the residuals of Eqs. (1) and (5), and the resulting set of equations is solved by means of the onestep Newton's method. To provide an accurate approximation for nodal values of the velocity and free surface coefficients, at time $t^{n}$, the second order Adams-Bashforth predictor is used; pressures are initialized with values corresponding to the previous time step. The predictor also provides an estimate of the time discretization error which is controlled with the time step size, and is kept small enough to obtain convergence in just one Newton iteration. Two time steps are computed, one is based on the norm of the velocity and the other on the norm of the free surface coefficients, the smallest one is chosen in the calculations.

The criteria adopted to select the appropriate finite element mesh were based on the following physical aspects of the problem.

(1) The motion of the fluid is more intense near the free surface, where larger gradients of the velocity occur. Therefore, nodes are concentrated at the free surface and its vicinity.

(2) A boundary layer is developed in the liquid just above the solid wall; consequently, the mesh is refined in this region.

(3) The number of elements in the $x$-direction turns on the wave number $(\alpha)$ and on the parameter $F$.

We next exemplify the type of numerical experiments carried out to determine an appropriate mesh when nonlinear 

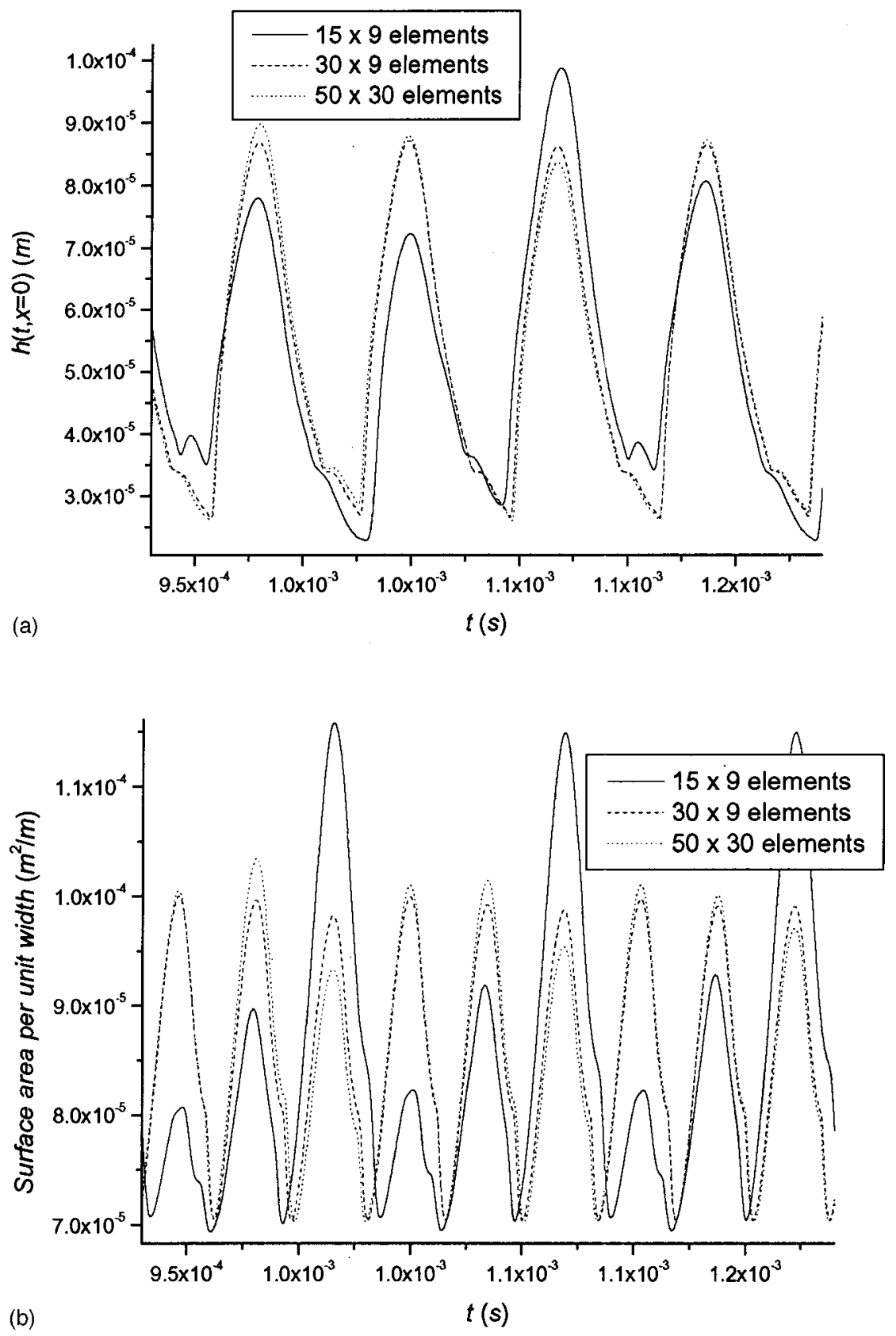

FIG. 2. (a) Predictions of the time evolution of one end of the free surface for point labeled $c$ in Fig. 3, computed with three different meshes. (b) Predictions of the time evolution of the interfacial area for point labeled $c$ in Fig. 3, computed with three different meshes. effects are important, that is for large values of the external amplitude. They correspond to points labeled $c$ and $d$ in Fig. 3 but they are applicable to any of the solutions presented in this work. We employed the following three meshes: $N E X$ $=15$ and $N E Y=9, N E X=30$ and $N E Y=9, N E X=50$ and $N E Y=30$. In all these meshes the relationship between the number of elements in the $x$ and $y$ direction was selected taking into account the aspect ratio of the computational domain, in order not to have very distorted elements.

In Figs. 2(a) and 2(b) we illustrate the evolution in time of the numerical solutions corresponding to point $c$ in Fig. 3 . We see that the three meshes predict the existence of a periodic state although the maximum amplitude attained by the free surface is clearly variable when the coarsest grid is employed [see Fig. 2(a)]; differences in the evolution of the free surface are slightly noticeable when the solutions computed with the other two meshes are compared. The same conclu- sion can be inferred from results depicted in Fig. 2(b) where the evolution of the interfacial area is illustrated.

To determine if this mesh was suitable to accurately predict not only periodic solutions but also the conditions under which the amplitude initially imposed to the free surface increases without bounds, we performed similar numerical tests for points located in Region 1-I of the stability maps; one of those points (labeled $d$ ) is shown in Fig. 3. Results that are not illustrated here, show that with none of these meshes a periodic solution is obtained, being the evolution of the free surface computed with the three meshes very similar.

From the above numerical experiments, we conclude that the mesh $N E X=30$ and $N E Y=9$ is suitable to follow the time evolution of the system when the initial liquid depth is equal to $5 \times 10^{-5} \mathrm{~m}$ even when nonlinear effects are important. A more refined mesh would only lead to an unnecessary increase of the CPU time. 


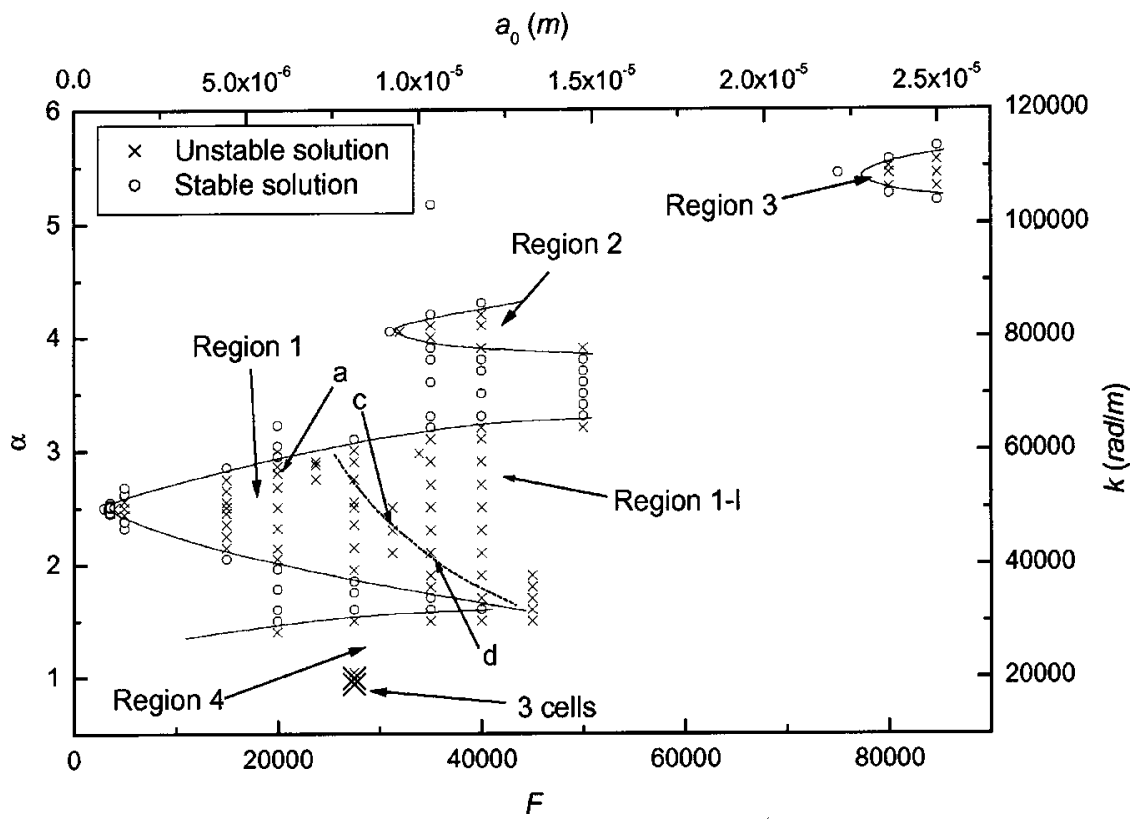

FIG. 3. Stability chart in the $F-\alpha\left(a_{0}-k\right)$ plane for $H_{0}=5$ $\times 10^{-5} \mathrm{~m}$.

\section{NUMERICAL RESULTS}

In order to determine the influence of the film thickness on the stability of liquid layers under forcing vertical oscillations, we have carried out computations with equilibrium depths between $10^{-4} \mathrm{~m}$ and $10^{-5} \mathrm{~m}$. The physicochemical properties correspond to water at $20^{\circ} \mathrm{C}$; thus, we have, $\rho$ $=10^{3} \mathrm{~kg} / \mathrm{m}^{3}, \sigma=70 \times 10^{-3} \mathrm{~N} / \mathrm{m}$, and $\mu=10^{-3}$ Pa s. The oscillation frequency of the container $(f)$ was set at $29 \mathrm{kHz}$ while its amplitude was widely varied.

In this section we first discuss the stability charts of the system and then we analyze the influence of the initial film thickness $\left(H_{0}\right)$ on the velocity fields.

\section{A. The stability maps}

The numerical solutions pertaining to each initial liquid depth give rise to a stability chart on the $F$ - $\alpha$ plane. In Figs. 3,4 , and 5 the charts for $H_{0}$ equal to: $5 \times 10^{-5} \mathrm{~m}, 2.27$ $\times 10^{-5} \mathrm{~m}$, and $10^{-5} \mathrm{~m}$, respectively, are illustrated. These maps and those obtained for the other equilibrium heights considered in this work, not shown here, are qualitatively similar; therefore, we first give a detailed description of the chart corresponding to $H_{0}=5 \times 10^{-5} \mathrm{~m}$ and then we point out the main differences observed when this variable is modified.

Every point of the stability maps represents the outcome

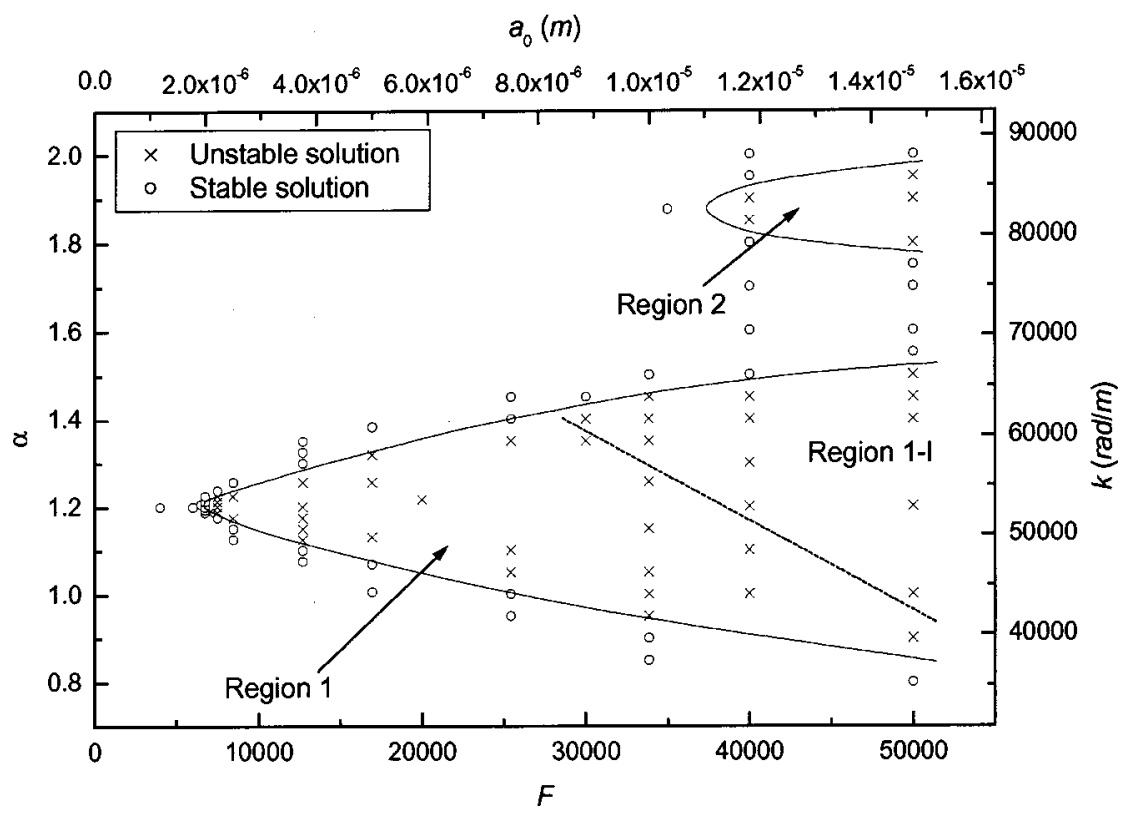

FIG. 4. Stability chart in the $F-\alpha\left(a_{0}-k\right) \quad$ plane for $H_{0}=2.27$ $\times 10^{-5} \mathrm{~m}$ 


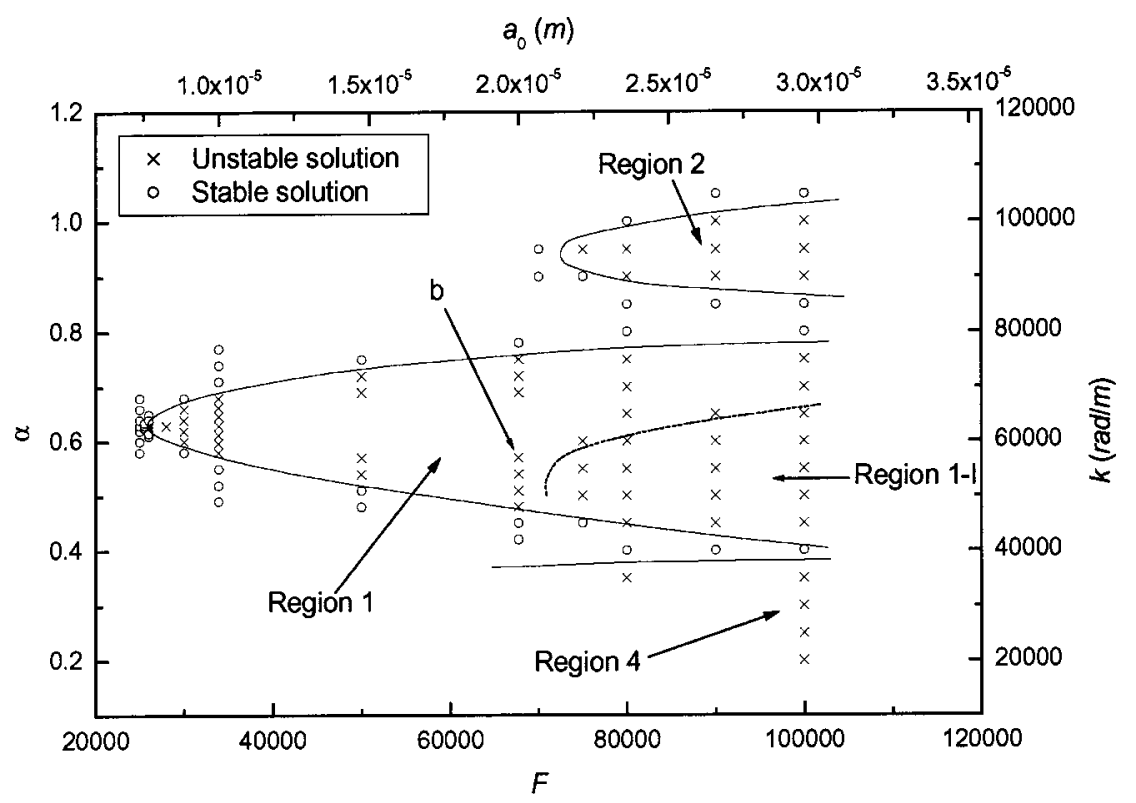

FIG. 5. Stability chart in the $F-\alpha\left(a_{0}\right.$ $-k$ ) plane for $H_{0}=10^{-5} \mathrm{~m}$.

of the time evolution of a rather small initial perturbation imposed to the motionless liquid. Circles indicate stable situations characterized by perturbations whose amplitudes decay in time, while crosses represent unstable cases characterized by perturbations whose amplitudes increase without bounds or that evolve toward time periodic solutions. To facilitate the analysis of the results each point drawn in Figs. 3 to 5 may be regarded either as a point on the $(F-\alpha)$ plane or on the $\left(a_{0}-k\right)$ plane.

It is easy to notice that the data reported in Fig. 3 can be grouped into four different regions identified as 1, 2, 3, and 4; the same situation could be detected in Figs. 4 and 5 although in these cases due to the narrower range of wave numbers investigated, we did not explore points pertaining to region 3 . In regions $1-3$, the relation existing between the dominant frequency of the wave appearing on the free surface and the frequency imposed on the motion of the container is equal to $1 / 2,1$ and $3 / 2$, respectively and in all of them only one elementary cell is observed. Region 4 presents subharmonic resonance (the free surface vibrates with a frequency equal to one half of the applied external frequency) but two elementary cells are formed. Since a reduction in the dimensionless wave number, $\alpha$, implies a proportional increase of the domain length, the points located here should probably be a duplication of points lying in region 1, a hypothesis that will be confirmed later. Also, we have detected three subharmonic elementary cells when the wave number was further reduced. Although other unstable regions can be found for larger values of $\alpha$, we have not investigated them because they are associated with too high external forces and, consequently, they have not practical interest.

Results illustrated in Fig. 3 show that regions 1 and 4 overlap for values of $F>45000$ but the other regions remain separated from one another in agreement with the linear stability analysis reported by Benjamin and Ursell. ${ }^{2}$

The instability threshold in each region is characterized by a single pair of values of $F$ and $\alpha$ : $\left(F_{C}=a_{C} \omega^{2} / g, \alpha_{C}\right)$; if $F$ goes beyond the critical value disturbances with a wave number within a specific range will be excited. That is, the resonance phenomenon is first observed when there is a fine tuning between the natural frequency of the system and the frequency of the imposed motion.

The wave number at the instability threshold increases as one moves from region 1 to region 3; also, the applied force required to attain that threshold increases in the same way suggesting that larger dissipation effects associated to stronger spatial gradients may be responsible for the higher values of $F$ needed to destabilize the system.

The four regions illustrated in Fig. 3 can be related to the theoretical work presented by Benjamin and $\mathrm{Ursell}^{2}$ for an ideal fluid. In their formulation the position of the interface is described by a series whose coefficients $c_{m}(t)$ satisfy the Mathieu equation, that is

$$
\frac{d^{2} c_{m}}{d T^{2}}+\left[p_{m}-2 q_{m} \cos 2 T\right] c_{m}=0,
$$

where $T=\omega t / 2$. It is well known that the stability of the solutions of this equation depends on the values of the parameters $p$ and $q$ that for the $m$ mode are defined by the following expressions (Eq. 2-13 in Ref. 2),

$$
\begin{aligned}
& p_{m}=\frac{\omega_{m}^{2}}{(\omega / 2)^{2}}, \quad \omega_{m}^{2}=k_{m} \tanh \left(k_{m} H_{0}\right)\left(g+\frac{k_{m}^{2} \sigma}{\rho}\right), \\
& q_{m}=2 k_{m} a_{0} \tanh \left(k_{m} H_{0}\right),
\end{aligned}
$$

where $\omega_{m}$ is the natural frequency of mode $m$ and $k_{m}$ $=m k$. For all points located in regions 1 to 3 of the stability map shown in Fig. 3, one elementary cell is observed; then, we employed $m=1$ in Eqs. (13) and (14) to evaluate $p$ and $q$ there, while for those points located in region 4 where two elementary cells are formed, we put $m=2$. The resulting points $(p, q)$ are illustrated in Fig. 6, where again crosses and circles represent unstable and stable solutions, respectively. Additionally, the boundaries of the regions predicted by the linear theory are shown. 


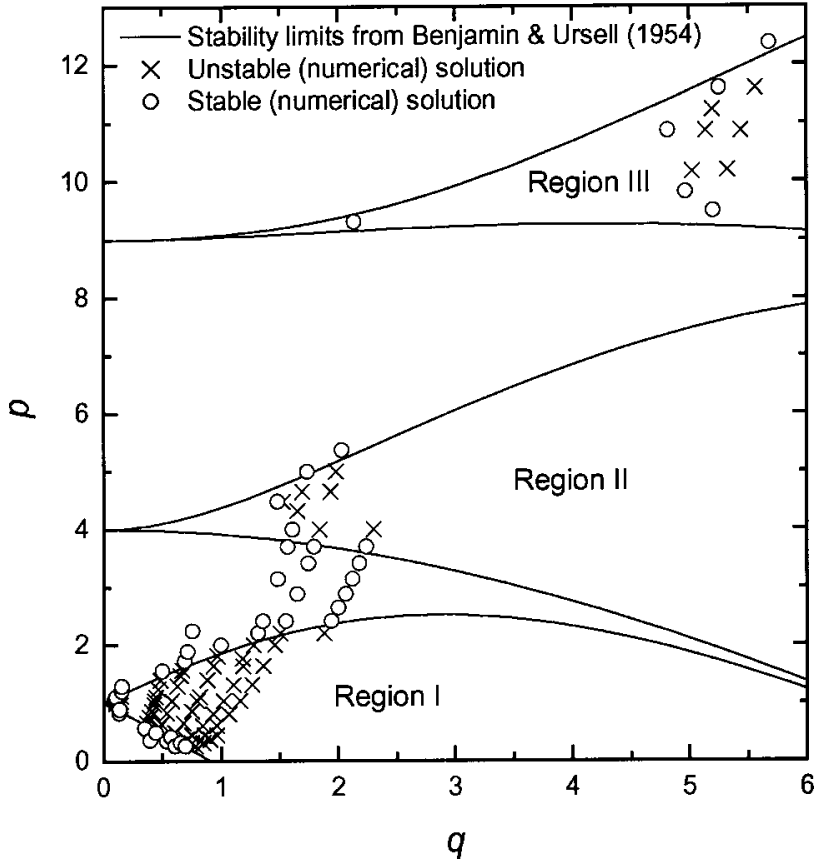

FIG. 6. Comparison of numerical results with the linear theory by Benjamin and Ursell.

It is clear that our results representing unstable evolutions of the free surface are located within the three regions predicted by the linear stability theory. Nevertheless, the stability limits are translated toward values of $q>0$. The parameters $F$ and $q$ have similar meaning because both depend on the amplitude of the external vibration. Then, we can conclude that when the viscous effects are considered, a fine tuning between the frequency of the imposed force and the natural frequency of the system is not enough to produce resonance; it is also required that $F$ be larger than a critical value. These results are in agreement with the theoretical analysis reported by Kumar and Tuckerman ${ }^{8}$ and by Cerda and Tirapegui ${ }^{9}$ for a slightly viscous fluid.

When points located in region 4 are mapped into the $(p-q)$ plane, they all lie in region 1 of Fig. 6, confirming the hypothesis that region 4 is a replication of region 1. A careful inspection performed of the corresponding numerical solutions have shown that this is indeed the case.

In order to detect the influence of the film thickness on the onset of the instability, we have mapped the stable points located close to the limits of region 1 in Figs. 3, 4 and 5 into the $(p, q)$ plane using Eqs. (13) and (14); the result is depicted in Fig. 7. As it is expected, the extension of the unstable region is reduced, not only because the threshold is shifted toward larger values of $q$ (that is, larger amplitudes of the imposed force) but also because the lower limit is shifted toward larger values of $p$, as $H_{0}$ is reduced.

With the purpose of validating the numerical solutions, we have solved the viscous model reported by Kumar and Tuckerman ${ }^{8}$ for the same initial liquid depths considered in Figs. 3 to 5. The values of $a_{0}$ obtained for each wave number $\alpha$ selected were then mapped into the $(p, q)$ plane using Eqs. (13) and (14), and finally the stability limits for the subharmonic region corresponding to each $H_{0}$ were illustrated in

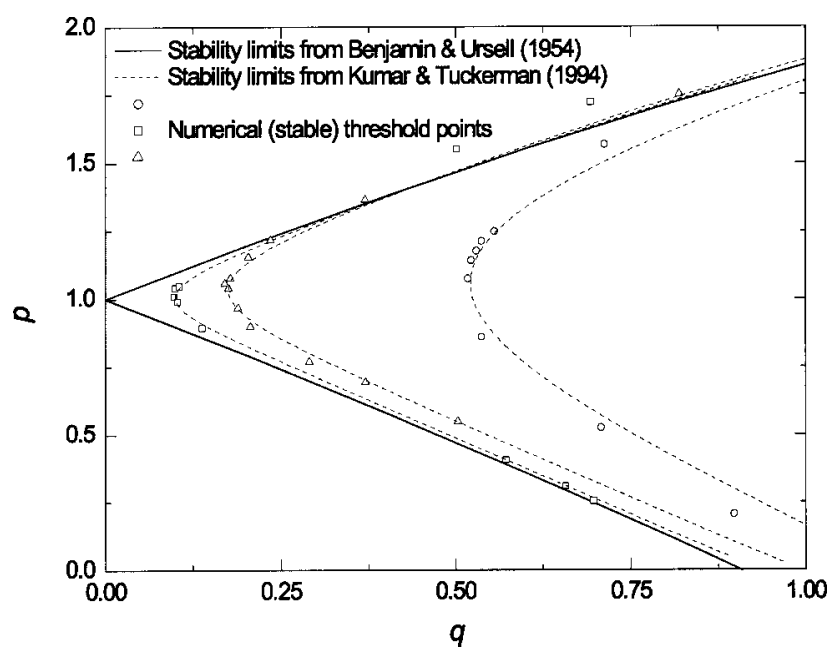

FIG. 7. Stability limits for selected values of $H_{0}$ near the threshold of region 1 (Figs. 3-5) and comparison of numerical results with Kumar and Tuckerman's model.

dashed lines in Fig. 7. It is evident that the agreement between our numerical solutions and those calculated with Kumar and Tuckerman's model is excellent. That is, the numerical solution accurately reproduces the boundaries of the unstable regions predicted by the linear stability analysis of the full hydrodynamic problem.

One of the practical applications of the problem under study is the production of sprays. Drops are formed from the crests of high capillary waves. Therefore, it would be useful to investigate the condition under which the amplitude of the free surface waves increases without bounds. With this purpose, we have closely examined the numerical solutions located in region 1 of the stability charts presented in Figs. 3-5 and we have delimited the subregions labeled 1-I, which are approximately defined by dashed-lines in the maps. For the points inside these zones our numerical predictions showjust before the computation breaks down-almost chaotic free surface evolutions and wave amplitudes increasing without bounds, suggesting that these points correspond to values of the parameters in which the atomization of the liquid might occur. Obviously, these solutions cannot be detected with simpler models based on the assumption of small free surface deformations.

An interesting feature of the results obtained is that the location of the subregion 1-I depends on the film thickness. In fact, for the larger values of $H_{0}$ considered in this work, this zone is located near the upper bound of region 1 (see Figs. 3 and 4), while for the thinnest value of the liquid layer it is located near the lower bound of the subharmonic region (see Fig. 5). In the first case, our results agree with the experiments and the numerical solutions reported by Jiang et al. ${ }^{16}$ in a range of very small forcing frequencies (3.15$3.34 \mathrm{~Hz}$ ), that is, for a long wave.

To detect the origin of the differences induced by the initial film thickness on the location of subregion 1-I, we have analyzed some of the solutions that are located near the boundary of this zone when the initial liquid depth is equal to $5 \times 10^{-5} \mathrm{~m}$ (Fig. 3) and $10^{-5} \mathrm{~m}$ (Fig. 5). These solutions are 


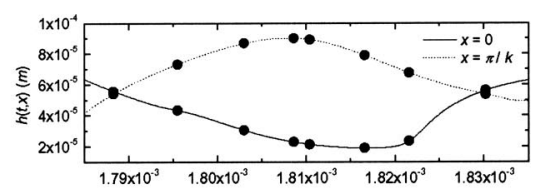

a: $H_{0}=5 \times 10^{-5} \mathrm{~m}, F=20000, \alpha=2.8$

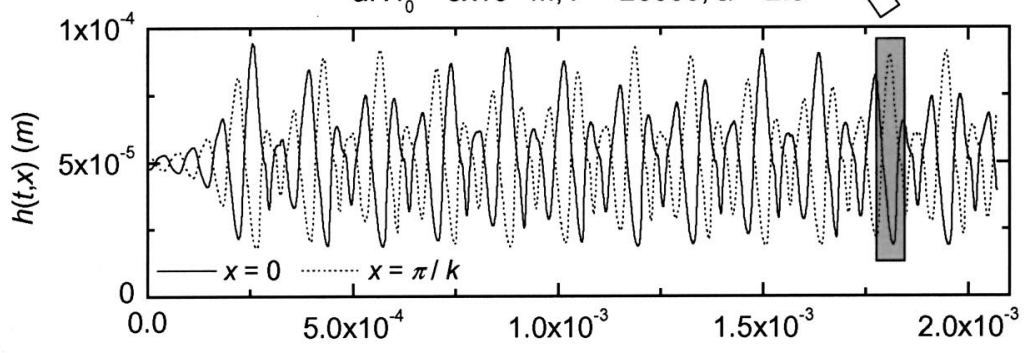

(a)

$t(s)$

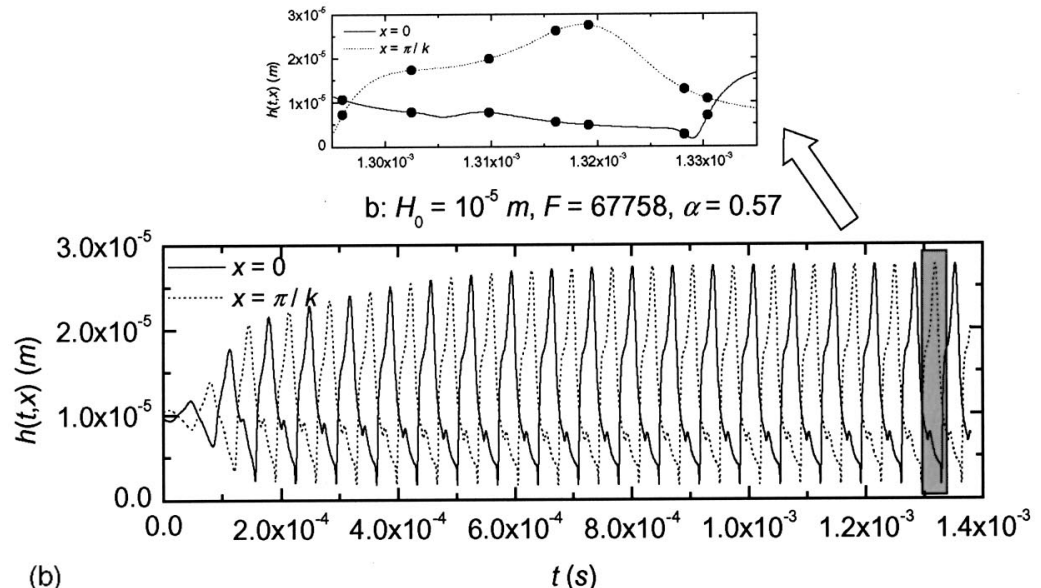

FIG. 8. Prediction of the time evolution of the film thickness at $x=0$ and $x=1$ for (a) point labeled $a$ in Fig. 3 and (b) point labeled $b$ in Fig. 5. for a fixed value of $F$, and for $\alpha$ varying within the range delimited by the lower and upper branches of region 1 in the corresponding stability map. We took $F$ equal to 20000 and 67759 , for $H_{0}=5 \times 10^{-5} \mathrm{~m}$ and $H_{0}=10^{-5} \mathrm{~m}$, respectively.

The examination of the free surface for the thicker liquid layer $\left(H_{0}=5 \times 10^{-5} \mathrm{~m}\right)$ once a time-periodic solution is developed for each value of $\alpha$, shows that the amplitude of the wave monotonically increases as the wave number is augmented, almost until the upper branch of the neutral stability curve is reached; in fact, from our numerical solutions not presented here, we found that the wave amplitude increases from $1.3 \times 10^{-5} \mathrm{~m}$, when $\alpha=2.05$, to $7.38 \times 10^{-5} \mathrm{~m}$ for $\alpha=2.86$.

When the same analysis is carried out for $H_{0}=10^{-5} \mathrm{~m}$ and $F=67759$, the amplitude of the wave corresponding to the time periodic-state achieved by the system at each selected value of $\alpha$, first increases and then diminishes as the dimensionless wave number is augmented, as in the previous case; nevertheless, according to our numerical solutions, the maximum amplitude computed is approximately equal to $2.65 \times 10^{-5} \mathrm{~m}$ and corresponds to $\alpha \approx 0.57$ (point labeled $b$ in Fig. 5), a value closer to the lower bound of region 1.

To illustrate the relevant features of the solutions developed near the onset of region $1-\mathrm{I}$, for $H_{0}$ equal to 5 $\times 10^{-5} \mathrm{~m}$ and $10^{-5} \mathrm{~m}$, we have depicted for the points la- beled $a$ and $b$ in Figs. 3 and 5, respectively, the time evolution of the free surface height at both ends of the computational domain, i.e., at the points that initially were the crest and the trough of the wave [Figs. 8(a) and 8(b)]. In Figs. 9(a) and 9 (b) we present the corresponding results of transforming the waveforms into Fourier space using the fast Fourier transform (FFT).

Results illustrated in Fig. 8 show that the amplitude of the free surface waves increase from the perturbations initially imposed up to certain final values in the two cases considered. When $H_{0}=10^{-5} \mathrm{~m}$ a time periodic-state is rapidly attained, while for $H_{0}=5 \times 10^{-5} \mathrm{~m}$ a repeated pattern is more difficult to detect. The results of the FFT analysis presented in Figs. 9(a) and 9(b), confirm that in both cases the dominant mode has a frequency equal to $(1 / 2) f$; however, the spectra for point $a$ is not as well defined as that for point $b$. This fact added to the relatively important peak that appears at a frequency equal to $(1 / 4) f$, contribute to the irregular aspect of the evolution of the amplitude of the free surface observed in the first case [see Fig. 8(a)].

The FFT analysis carried out at point $b$ reveals the presence of smaller peaks at frequencies equal to multiples of $f: f$, $(3 / 2) f, 2 f, \ldots$; that is, higher harmonics are also excited and they are responsible for the departure of the waveform from a sinusoidal curve [see Fig. 8(b)]. 
a: $H_{0}=5 \times 10^{-5} m, F=20000, \alpha=2.8$

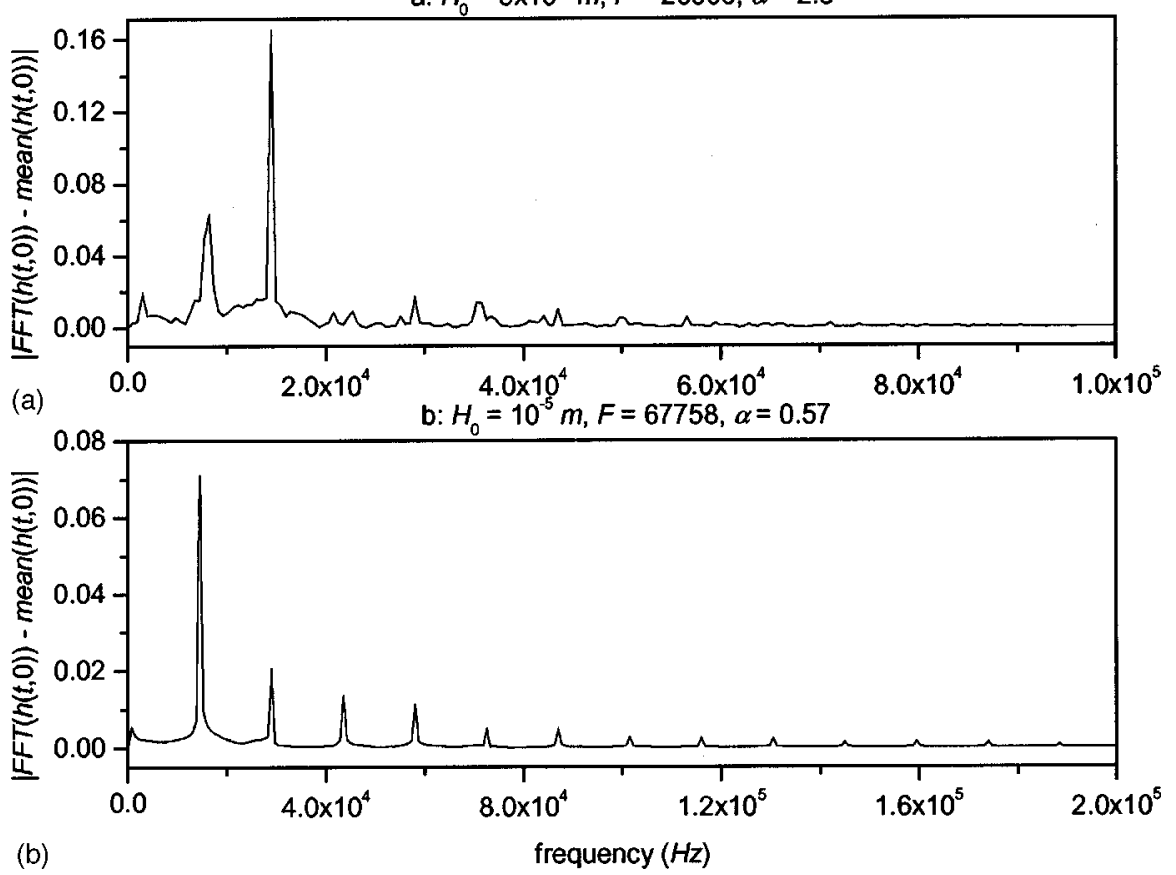

FIG. 9. (a) and (b) Discrete Fourier transform of the temporal evolution at $x=0$ for points $a$ and $b$, respectively.

In order to detect the possible mechanisms involved in the breaking of the free surface waves for a large $\left(H_{0}=5\right.$ $\left.\times 10^{-5} \mathrm{~m}\right)$ and a very thin $\left(H_{0}=10^{-5} \mathrm{~m}\right)$ liquid layer, we have examined the evolution of the free surface shapes during a short time interval (about half cycle) for cases $a$ and $b$ (Fig. 10). The intervals chosen are enclosed by a rectangle in Figs. 8(a) and 8(b), and the times selected are indicated in the insets of these illustrations where an enlarged view of the framed regions is shown. The shapes corresponding to point $a(1-8)$ are drawn with solid line while those for point $b$ (9-15) are depicted in dashed line.

It is easy to see that the more remarkable difference between the curves corresponding to these two cases, is the steepness of the wave that is the ratio between the peak to peak amplitude and the wavelength; in fact, this quantity is approximately equal to 0.65 for $H_{0}=5 \times 10^{-5} \mathrm{~m}$ (point $a$ ), and to 0.24 for $H_{0}=10^{-5} \mathrm{~m}$ (point $b$ ).

The profiles corresponding to point $a$, show that as the right side of the domain moves upward a large quantity of liquid is displaced toward the crest giving rise to a high peak, where the radius of curvature of the interface becomes small and, consequently the capillary forces become large. Considering that an increase of the amplitude of the external force will produce a higher and more peaked wave, these results suggest that drops might be formed at the maximum crest elevation. It is interesting to note that, due to the presence of higher order harmonics, the free surface never becomes horizontal during the time interval.

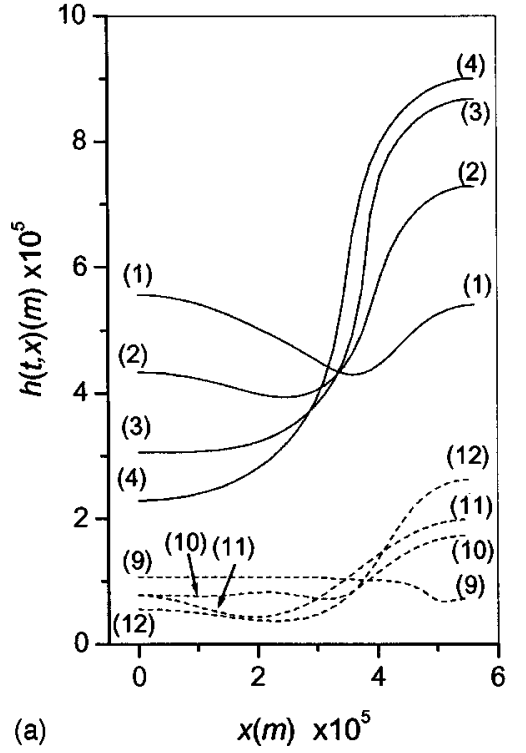

(a)

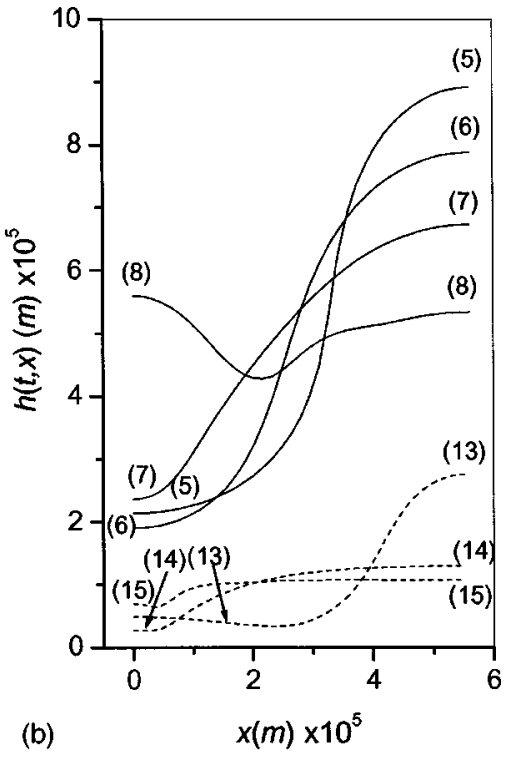

FIG. 10. Predicted free surface shapes for selected times corresponding to the half cycle enclosed with dashed lines in Figs. 8(a) and 8(b). The curves drawn in solid lines (1-8) are for $H_{0}=5 \times 10^{-5} \mathrm{~m}$ and those depicted in dashed lines $(9-15)$ are for $H_{0}=1 \times 10^{-5} \mathrm{~m}$; the curves are numbered sequentially for increasing values of time. 


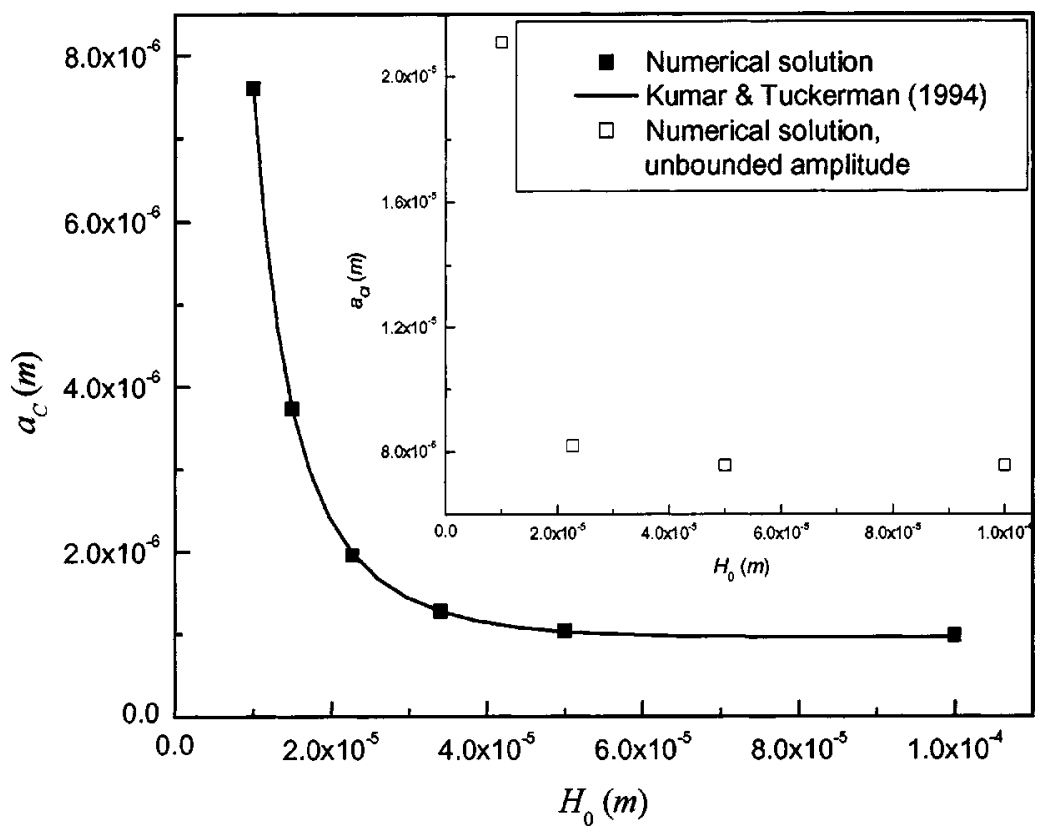

FIG. 11. Critical amplitudes vs $H_{0}$ for region $1\left(a_{C}\right)$ and for subregion 1-I $\left(a_{C I}\right)$ (inset).
If we now consider the sequence of wave shapes illustrated in dashed lines for $H_{0}=10^{-5} \mathrm{~m}$, we see that the influence of the bottom boundary on the evolution of the system becomes very important. In fact, in this case, a very thin liquid film is formed during the evolution (the minimum liquid height at the trough of the wave is smaller than 3 $\times 10^{-6} \mathrm{~m}$ in curve 14 ). As the height of the liquid located at the right of the domain increases, a small wave travels toward the left; therefore, the free surface presents a depression separating two regions: one where there is a large amount of liquid and the other where a thin liquid film exists; the latter will resist the leveling of the liquid layer once the highest liquid elevation is reached at the other end of the domain. In this case, an increase of the amplitude of the external vibration should produce a larger crest and a thinner film, a situation that might favor the film disruption instead of the ejection of drops.

The large magnitude of the capillary forces compared to the effective gravity forces, is responsible for the rounded crests presented by the waves during the cycle; that is, on the verge of the numerical break down of our solutions we do not see the flat crests observed-for low excitation frequencies and when the capillary effects are much lower-by Jiang et al. ${ }^{16}$

Finally, in the two cases analyzed, the temporal symmetry is broken, a result that can also be inferred from Figs. 8(a) and 8 (b).

Results depicted in Figs. 3, 4, and 5 and those not reported here for other selected values of $H_{0}$, show that the stability charts are qualitatively similar. Indeed, the same regions previously described are detected and, at the onset of the instability, the interface is always subharmonically excited with a frequency equal to one half the frequency of the external vibration; however, the following differences can be observed.

(1) The minimum value of $F$ required to produce a wavy interface, which in our case corresponds to a minimum in forcing amplitude $\left(a_{C}\right)$ since the applied frequency is kept constant, increases as the depth of the liquid layer decreases; that is, the system becomes more stable if viscous effects are relatively larger. Also the minimum value of $F$ associated to the rupture of the free surface increases as the depth of the liquid layer is reduced.

(2) The wave number $k=k_{C}$ of the elementary cell developed at the onset of the instability increases as the film thickness diminishes.

Next, we discuss both differences in detail.

\section{B. Critical value of $a_{0}$ as a function of $H_{0}$}

In Fig. 11, the value of the critical amplitude $\left(a_{C}\right)$ is illustrated as a function of $H_{0}$ at the threshold of the first subharmonic region (region 1 in Figs. 3-5). It is readily seen from our numerical predictions that larger amplitudes of the external force are required to destabilize a system as the initial liquid height is reduced; also, the effect of the film thickness becomes important for $H_{0} \leqslant 5 \times 10^{-5} \mathrm{~m}$. In fact, if the depth of the liquid layer is reduced below this value, the external force will have to be greatly augmented to turn the system unstable, while it remains almost constant for $H_{0}$ larger than $5 \times 10^{-5} \mathrm{~m}$.

It is of interest to compare our values of critical wave amplitude with those calculated with the exact solution of the linear viscous hydrodynamic problem reported by Kumar and Tuckerman. ${ }^{8}$ Therefore, we have also depicted in Fig. 11 the minimum amplitude required to destabilize the system as a function of the initial liquid depth obtained by solving that problem. It is easy to see that the agreement is excellent; in fact, the largest difference observed is below $2.5 \%$ and it corresponds to $H_{0}=10^{-4} \mathrm{~m}$.

The open squares depicted in the inset of Fig. 11, represent the minimum forcing amplitude $\left(a_{0}=a_{C I}\right)$ required to produce a solution in region 1-I for four values of the initial liquid depth considered in this work. As it is expected, $a_{C I}$ 


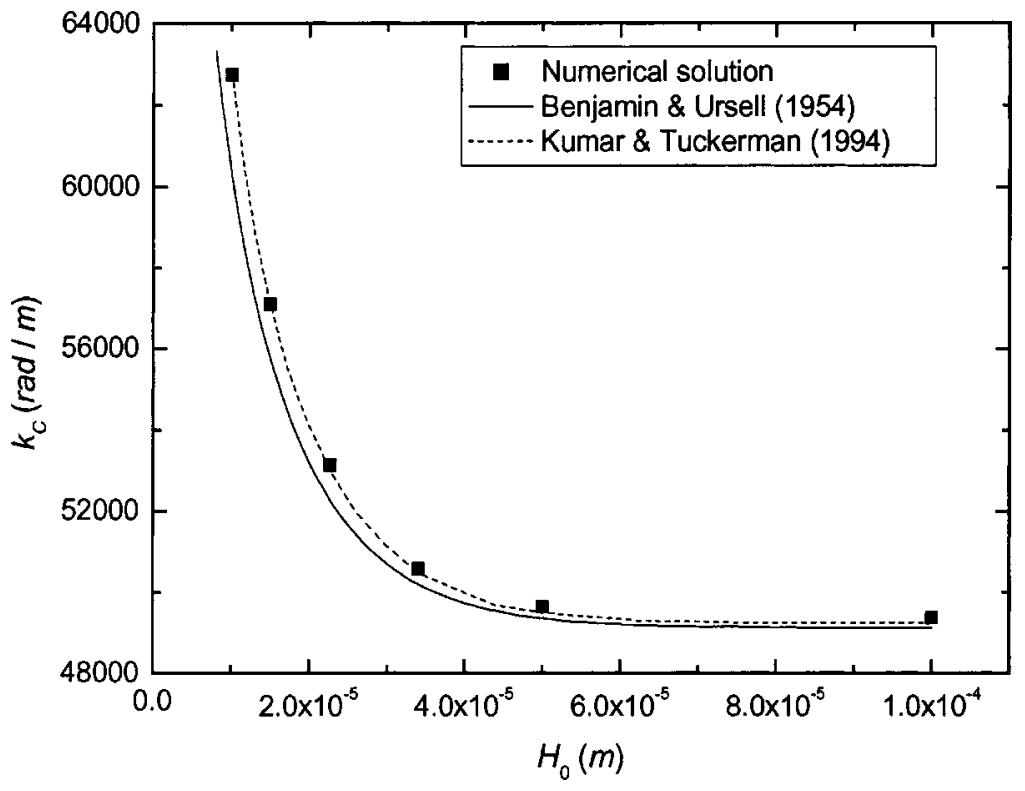

FIG. 12. Threshold values of $k$ vs $H_{0}$ for region 1 when $f=29 \mathrm{kHz}$. diminishes as $H_{0}$ is augmented; however, for $H_{0}>5$ $\times 10^{-5} \mathrm{~m}, a_{C I}$ becomes almost insensitive to changes in film thickness, this result confirms that the behavior of the system is no longer affected by the presence of the bottom wall.

As we have just mentioned, the onset of subregion 1-I occurs at larger amplitudes of the external force when $H_{0}$ diminishes; nevertheless, the ratio between the amplitude values at the onset of subregion 1-I and at the threshold of region 1 , i.e., $a_{C I} / a_{C}$, diminishes as the initial liquid depth decreases. It is easy to verify from the results reported in Fig. 11 , that this ratio is approximately equal to 7 and 3 for $H_{0}$ $=10^{-4} \mathrm{~m}$ and $H_{0}=10^{-5} \mathrm{~m}$, respectively.

Goodridge et al. ${ }^{17}$ in their experimental work on the prediction of the threshold amplitude for drop ejection, report that this value depends on the frequency of the external vibration imposed to the system. These authors found that in the case of water, the critical amplitude for the inception of drop ejection $\left(a_{D}\right)$ is given by $a_{D}=2.39\left(\sigma / \omega^{2} \rho\right)^{1 / 3}$; therefore, for the system analyzed in this work, $a_{D} \approx 3 \times 10^{-5} \mathrm{~m} \mathrm{a}$ value larger than those reported here for the onset of region $1-\mathrm{I}$ in the inset of Fig. 11.

\section{The critical value of the wave number as a function of $\boldsymbol{H}_{0}$}

In Fig. 12 the wave numbers of the elementary cells formed at the threshold of region $1, k=k_{C}$, are plotted as a function of the initial film thickness, the points there illustrated represent our numerical solutions while the continuous line corresponds to the inviscid solution, that is, $k_{C}$ is evaluated from

$$
\omega_{m}=\sqrt{\tanh \left(k_{m} H_{0}\right)\left(\frac{k_{m}^{3} \sigma}{\rho}+k_{m} g\right)},
$$

where $\omega_{m}=14500 \pi \mathrm{s}^{-1}$ and $m=1$; also, the values of $k_{C}$ calculated with Kumar and Tuckerman's model are shown in dashed lines. These results show that the influence of the bottom wall of the container becomes noticeable when the film thickness is smaller than $5 \times 10^{-5} \mathrm{~m}$. Thus, for larger values of the initial liquid depth the critical wave number remains almost constant, while if $H_{0}$ is below $5 \times 10^{-5} \mathrm{~m}$, $k_{C}$ rapidly increases as the initial film thickness diminishes. Although either in the inviscid or in the viscous case, the solid wall affects the flow through the normal boundary condition deviating the liquid in its vicinity, we see that the wave numbers of our full model are larger than those predicted by Benjamin and $\mathrm{Ursell}^{2}$ but, as the results illustrated show, they are in very good agreement with those evaluated with the model reported by Kumar and Tuckerman. ${ }^{8}$ In fact, these authors found that in the range of low viscosity, an increase of this property reduces the value predicted for the critical wavelength if viscous dissipation in the bulk is taken into account and viscous dissipation at the solid boundary is not considered. In the present case, we observe that as the initial film thickness decreases, the differences between the predictions of the complete model and those of the linear theory, increase; thus, viscous effects reduce the length of the elementary cell at which the resonance phenomenon is observed. Our numerical solutions show that this reduction is $0.57 \%$ when $H_{0}$ is equal to $5 \times 10^{-5} \mathrm{~m}$ and $3.9 \%$ when the initial depth is $10^{-5} \mathrm{~m}$.

\section{The evolution of the free surface and the velocity fields at the onset of the instability (region 1)}

In this section we analyze the influence of the initial liquid depth on the flow fields developed near the threshold. The two cases studied correspond to values of $H_{0}$ equal to $10^{-4} \mathrm{~m}$ and $10^{-5} \mathrm{~m}$, that are the maximum and minimum initial liquid depth considered in this work; the thresholds for these two cases were obtained at $F=3400, \alpha=4.95$, and $F$ $=26000, \alpha=0.63$.

In Figs. 13(a) and 14(a) we present the time evolutions of the $x$-points that initially are the trough of the wave for these two cases. Insets of these figures depict the interface location of the liquid layer at $x=0$ over approximately half 

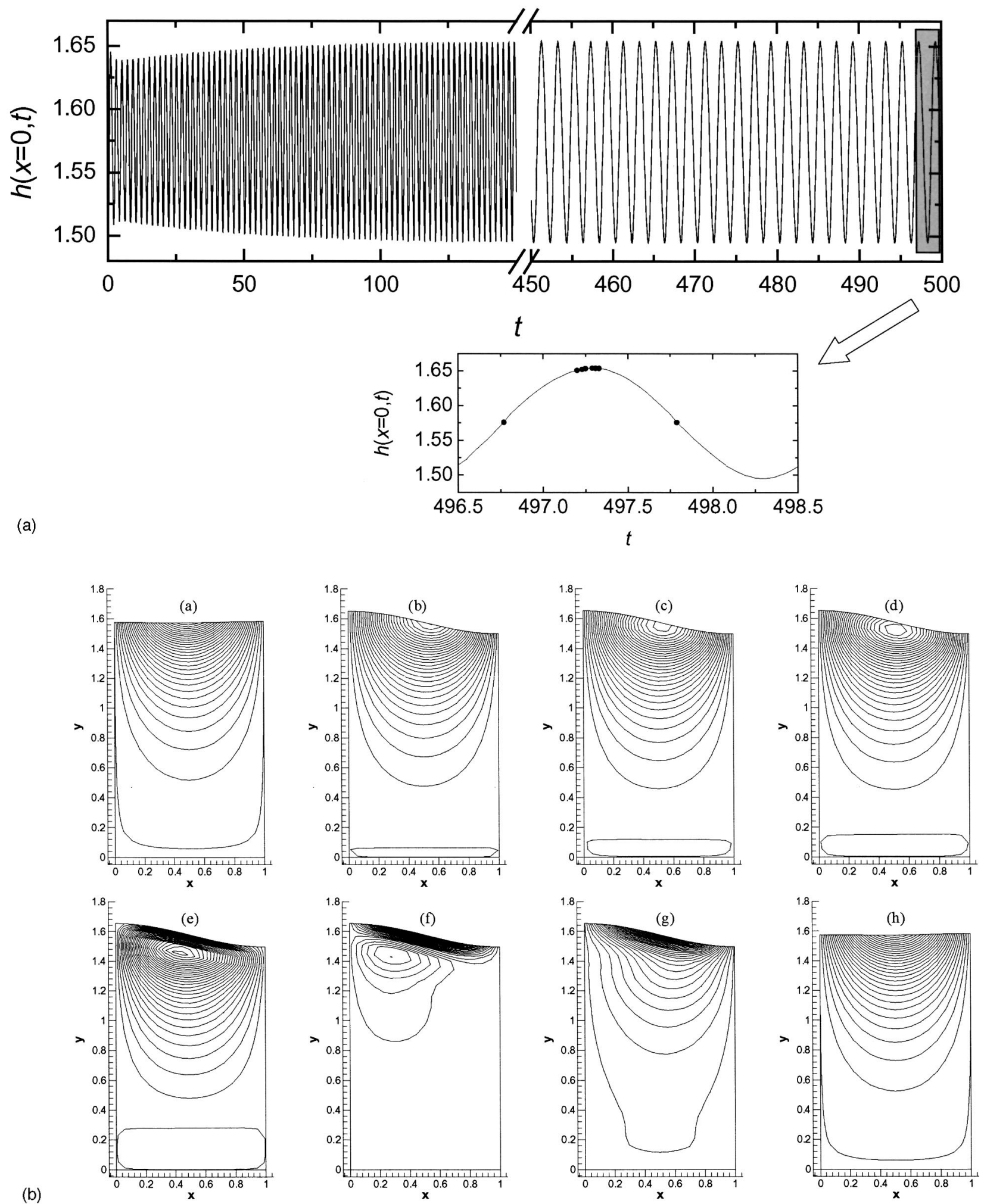

FIG. 13. (a) Temporal evolution of the film thickness at $x=0$ near the threshold of the first subharmonic region for $H_{0}=10^{-4} \mathrm{~m}$. (b) Streamlines corresponding to the selected times illustrated in the inset of (a); the figures are ordered alphabetically for increasing values of time.

cycle and the dots shown correspond to selected times at which the streamlines have been evaluated. The results are portrayed in Figs. 13(b) and 14(b) for $H_{0}$ equal to $10^{-4} \mathrm{~m}$ and $10^{-5} \mathrm{~m}$, respectively.

It is easy to notice [see Figs. 13(a) and 14(a)] that the wave amplitude at the free surface increases from the initially imposed perturbation until the constant value corresponding to the time periodic solution is achieved. A simple analysis based on the fast Fourier transform confirms that the period of the free surface oscillation is twice the period 

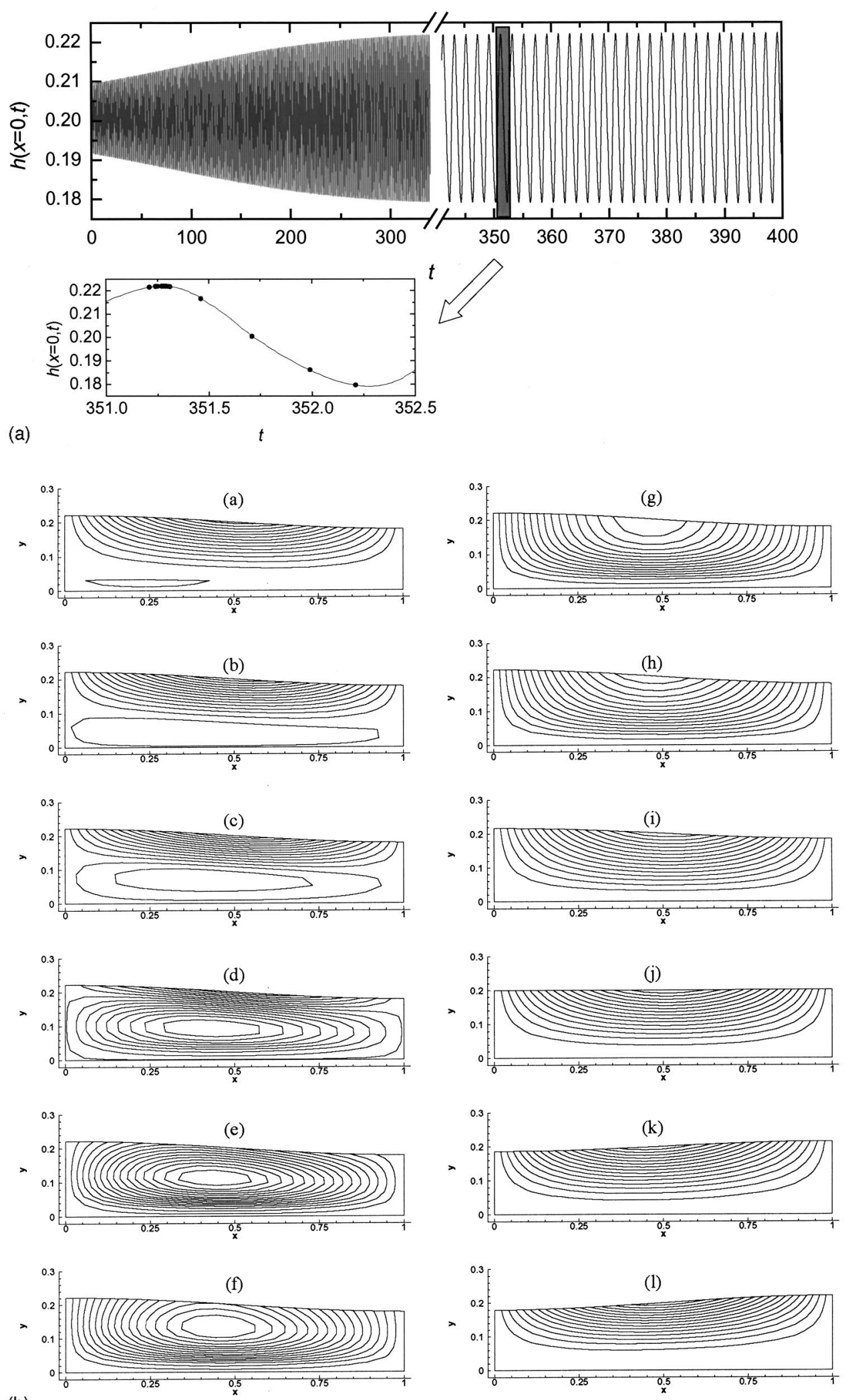

(b)

FIG. 14. (a) Temporal evolution of the film thickness at $x=0$ near the threshold of the first subharmonic region for $H_{0}=10^{-5} \mathrm{~m}$. (b) Streamlines corresponding to the selected times illustrated in the inset of (a); the figures are ordered alphabetically for increasing values of time.

of the external vibration; that is, the principal mode excited is subharmonic in agreement with experimental results (see, for instance, Hasegawa et al. ${ }^{18}$ ). Also, the shape of the wave is almost sinusoidal at the onset of the instability for all the values of $H_{0}$ considered in this work. This result contrasts with the result reported by Murakami and Chikano ${ }^{11}$ in their numerical study of this problem for a more viscous fluid (0.0072 Pa s) and at a much lower frequency of the external 
vibration $(41 \mathrm{~Hz})$, who found that the critical waveform is not sinusoidal. The analysis of the wave computed by these authors using the FFT, shows the presence of odd $(1 / 2 \omega, 3 /$ $2 \omega, 5 / 2 \omega, \ldots)$ and even components $(\omega, 2 \omega, 3 \omega, \ldots)$ being the coefficients of the first two odd frequencies-which are the largest - of the same order.

The streamlines illustrated in Fig. 13(b) for $H_{0}$ $=10^{-4} \mathrm{~m}$ correspond to the instants of time identified with dots in the inset of Fig. 13(a). The amplitude of the external force required to produce this motion is equal to 1.0036 $\times 10^{-6} \mathrm{~m}$, and as we have already mentioned the imposed force is approximately equal to the minimum needed to develop free surface waves with a frequency equal to $\omega / 2$; also the aspect ratio $\left(\Lambda=k H_{0} / \pi\right)$ of the elementary cell formed is nearly 1.57 , a value almost equal to the inviscid flow case.

The sequence of streamlines presented in the figure, show the existence of two swirls, one near the bottom of the container and the other at the free surface, both recirculations are present during a very short period of time. The swirl located on the solid wall is formed when the liquid is moving from right to left and the cell height is almost maximum at the left side. Under these conditions, the pressure on the plate at $x=0$ is larger than the pressure at $x=1$; therefore, the liquid located over the solid wall begins to move from left to right and a recirculating flow is developed. The size of this recirculation grows in time but is limited by the appearance of another swirl at the free surface, where the stronger motion takes place. After a very short period of time, all the fluid moves together, but only in the zone located near the free surface the modulus of the velocity is important. That is, the boundary layer that exists along the solid wall is very thin compared with $H_{0}$ and does not affect the dynamics of the system.

Figure 14(b) illustrates, near the instability threshold of region 1 , the evolution of the flow pattern during half cycle of a system with an initial liquid depth equal to $10^{-5} \mathrm{~m}$. The amplitude of the external force required to produce this motion is equal to $7.674 \times 10^{-6} \mathrm{~m}$ and the aspect ratio of the elementary cell developed is $\Lambda=0.200$; that is, a value slightly larger than the one corresponding to the ideal case (0.192).

The sequence of streamlines depicted in this figure shows that in this case there is only one swirl; in fact, the recirculation formed near the solid wall increases in size displacing the liquid above it, and finally involves the whole cell. That is, the effects of the viscous boundary layer that exists near the plate affect the motion of all the fluid, a situation completely different to that illustrated in Fig. 13(b) for $H_{0}=10^{-4} \mathrm{~m}$.

Although the analysis of the velocity fields associated to Faraday waves here presented is limited to two cases only, it shows that the streamlines developed for a slightly viscous fluid strongly depends on the viscous effects associated to the bottom wall. The vorticity generated at the solid boundary has almost negligible effects on the flow pattern when the liquid depth is large $\left(H_{0}=10^{-4} \mathrm{~m}\right)$; however, it affects a larger region as $H_{0}$ diminishes, and it might even involve the whole cell.

\section{CONCLUDING REMARKS}

The time evolution of thin liquid films subject to a periodic vertical oscillation, has been numerically analyzed by solving the Navier-Stokes equations for an incompressible liquid. The results portrayed pertain to a slightly viscous fluid; in fact, the values employed for the physicochemical properties of the liquid were those of water. From our numerical results we built charts delimiting instability regions in the $F$ - $\alpha$ plane. These regions nicely fit into the instability zones determined almost 50 years ago by Benjamin and Ursell for an ideal fluid; nonetheless, our results occupy these zones only partially, making evident the stabilizing influence of viscosity as previously reported by Kumar and Tuckerman and Cerda and Tirapegui. Moreover, the stability limits numerically determined are in excellent agreement with the limits of the linear stability analysis presented by Kumar and Tuckerman.

We have studied the effect of the thickness of the liquid film on Faraday's phenomenon. The results just presented confirm previous findings obtained by linear stability analysis; among them that stronger exciting forces are needed to produce unstable waves as the thickness of the film is reduced. Also, for the cases studied, that the lower boundary of the unstable regions in the $F$ - $\alpha$ charts appears to move toward higher wave number values (see Fig. 6).

To detect the amplitudes of the external vibration at which the breaking of the surface waves might take place, we have delimited a region of the stability maps in which the wavy motion of the free surface appears to increase without bounds. Although a more complete numerical analysis concerning the evolution of the free surface is required, our numerical solutions show evidences that when the initial liquid depth is very small, the instability process that produces the free surface disruption might be different from the drop ejection mechanism.

We have also confirmed, for the case under analysis, that a film thickness of about $5 \times 10^{-5} \mathrm{~m}$ delimits two zones of clear different behavior; that is, if the film thickness is larger than this value the force needed to turn the surface unstable is almost constant, and so is the length of the unstable wave appearing at the instability threshold. On the other hand, if the film is thinner than $5 \times 10^{-5} \mathrm{~m}$, the applied force at the instability threshold increases almost exponentially as the thickness of the film is reduced; accordingly, the length of the wave appearing at the onset of the instability is rapidly reduced. Since we suspected that the reasons behind this change of behavior dwelled on viscous effects originated at the solid wall, we studied in detail the time evolution of the flow fields for two systems with initial film thickness of $10^{-4} \mathrm{~m}$ and $10^{-5} \mathrm{~m}$, respectively; i.e. well above and below the limit value just mentioned.

When the film thickness is large $\left(H_{0}=10^{-4} \mathrm{~m}\right)$ the evolution of the streamlines within a period of oscillation evidences that the vorticity generated at the wall is weak and does not interact with the surface motion. In fact, the flow motion reverses by developing - at the interface-a thin boundary layer that rapidly grows and occupies the whole cell. Thus, as long as the flow in the vicinities of the solid 
wall does not influence the flow at the interface, the thickness of the film becomes irrelevant for the instability process, and the system is insensitive to changes in $H_{0}$.

A totally different situation occurs when the film thickness is small $\left(H_{0}=10^{-5} \mathrm{~m}\right)$; in this case the time evolution of the streamlines shows that the vorticity generated at the solid wall rapidly invades the cell reaching the free surface and reversing the fluid motion on it. Consequently, given this interaction, it seems reasonable that the instability process should strongly depend upon the value of the film thickness.

In this work we have solved the full Navier-Stokes equations without having recourse to the usually employed simplifications; e.g., Stokes flow assumption and the use of linear damping terms to account for viscous forces. The methodology employed allowed the construction of stability maps characterized by different equilibrium heights and the detection of the region where the breaking of the waves might occur; it also provides valuable information through the time evolution of the easily portrayed pressure and velocity fields. This last feature will be extensively exploited in a future work where the presence of surfactant will be considered.

\section{ACKNOWLEDGMENTS}

This research has been supported by the Universidad Nacional del Litoral, CONICET and the ANPCYT.

${ }^{1} \mathrm{M}$. Faraday, "On the forms and states assumed by fluids in contact with vibrating elastic surfaces," Philos. Trans. R. Soc. London 121, 319 (1831).

${ }^{2} \mathrm{~T}$. N. Benjamin and F. Ursell, "The stability of the plane free surface of a liquid in vertical periodic motion," Proc. R. Soc. London, Ser. A 225, 505 (1954).
${ }^{3}$ J. R. Ockendon and H. Ockendon, "Resonant surface waves," J. Fluid Mech. 59, 39 (1973).

${ }^{4}$ J. W. Miles, "Nonlinear surface waves in closed basins," J. Fluid Mech. 75, 419 (1976).

${ }^{5}$ J. W. Miles, "Surface-wave damping in closed basins," Proc. R. Soc. London, Ser. A 297, 459 (1967).

${ }^{6} \mathrm{~J}$. W. Miles and D. Henderson, "Parametrically forced surface waves," Annu. Rev. Fluid Mech. 22, 143 (1990).

${ }^{7}$ J. W. Miles, "On Faraday waves," J. Fluid Mech. 248, 671 (1993).

${ }^{8} \mathrm{~K}$. Kumar and L. Tuckerman, "Parametric instability of the interface between two fluids," J. Fluid Mech. 279, 49 (1994).

${ }^{9}$ E. A. Cerda and E. L. Tirapegui, "Faraday's instability in viscous fluid," J. Fluid Mech. 368, 195 (1998).

${ }^{10}$ J. Wright, S. Yon, and C. Pozrikidis, "Numerical studies of twodimensional Faraday oscillations of inviscid fluids," J. Fluid Mech. 402, 1 (2000).

${ }^{11}$ Y. Murakami and M. Chikano, "Two-dimensional direct numerical simulation of parametrically excited surface waves in viscous fluid," Phys. Fluids 13, 65 (2001).

${ }^{12}$ O. Lioubashevski, H. Arbell, and J. Fineberg, "Dissipative solitary states in driven surface waves," Phys. Rev. Lett. 76, 3959 (1996).

${ }^{13}$ H. S. Kheshgi and L. E. Scriven, "Penalty finite element analysis of unsteady free surface flows," in Finite Elements in Fluids, edited by R. H. Gallagher and J. T. Oden (Wiley, New York, 1984), p. 393.

${ }^{14}$ S. Kistler and L. E. Scriven, "Coating flow computations," in Computational Analysis of Polymer Processing, edited by J. R. A. Pearson and S. M. Richardson (Applied Science, London, 1983), p. 243.

${ }^{15}$ P. M. Gresho, R. L. Lee, and R. L. Sani, "On the time-dependent solution of the incompressible Navier-Stokes equations in two and three dimensions," in Recent Advances in Numerical Methods in Fluids, edited by C. Taylor and K. Morgan (Pineridge, Swansea, 1979), Vol. 1, p. 27.

${ }^{16}$ L. Jiang, C. L. Ting, M. Perlin, and W. Schultz, "Moderate and steep Faraday waves: Instabilities, modulation and temporal asymmetries," J. Fluid Mech. 329, 275 (1996).

${ }^{17}$ C. L. Goodridge, W. T. Shi, and D. P. Lathrop, "Threshold dynamics of singular gravity-capillary waves," Phys. Rev. Lett. 76, 1824 (1996).

${ }^{18}$ E. Hasegawa, T. Umehara, and M. Atsumi, "The critical condition for the onset of waves on the free surface of a horizontal liquid layer under a vertical oscillation," Bull. JSME 27, 1625 (1984). 